\title{
Designing an Efficient Method for Measuring the Permittivity of Samples in a Various Frequency Range using Capacitive Sensor
}

\author{
${ }^{1}$ Kalyan Sagar Kadali and ${ }^{2} \mathrm{~L}$. Rajaji \\ ${ }^{1}$ Department of EEEM, AMET University, Chennai, India \\ ${ }^{2}$ Department of Electrical and Electronics Engineering, \\ ARM College of Engineering and Technology, Chennai, India
}

\begin{abstract}
Material can be interacted by energy called electromagnetic radiation. For characterizing medium of the material, the process of interaction plays a major role in all application which are employed for sensing. Characteristics of any material can be represented by constant dielectric parameters. They can be identified in various conditions of environment by emitting, interacting and receiving the electromagnetic radiation. Here a system is proposed for developing an effective method measuring the permittivity in the different types of liquid samples like salt water, tap water and in the mineral water at various ranges of frequencies at certain temperature by using capacitive sensor and temperature sensor.
\end{abstract}

Key words: Permittivity measurement, wide frequency range, capacitive sensor, frequencies, sensor, environment

\section{INTRODUCTION}

An extensive role in several areas in industry sectors and scientific research were played by dielectric materials. Due to the various developments over semiconductor technology, various IC industries design the miniaturized components. In recent times capacitive sensors have been widely used in several sensing applications. They are measurement of pressure, testing the level of liquid, measuring the thickness of dielectric, sections by capacitive imaging through any kind of wave which are penetrating through it and measuring the displacements. These sensors can be developed in macro and micro form of structures. So, it can play a vital role in the technology of micro sensors.

The permittivity of water can be measured by using different methods but the most important method is a capacitive method by measuring the capacitance. Proposed system consists of a probe of capacitance and the measurement module for monitoring the change of capacitance. The module for measuring contains capacitive sensor module and temperature sensor module which are used in a different way. The electrical conductivity is the very important quantities where the complex permittivity on water is affected. It is linked to the made-up part of complex permittivity. Conductivity effects on the measurement of capacitance in liquids by employing cylindrical capacitive sensor. Frequency is another parameter in that are affecting complex

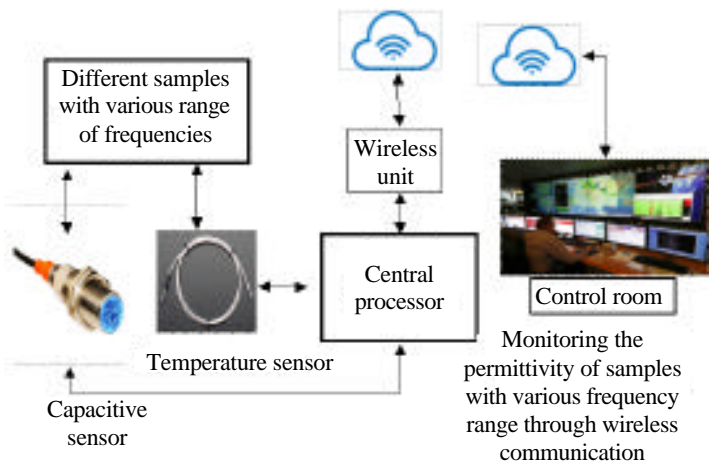

Fig. 1: System architecture for designing an efficient method of measuring the permittivity of samples in a various frequency range using capacitive sensor

permittivity (Blackham and Pollard, 1997; Krupka et al., 1998; Ellison et al., 1998; Huang et al., 1989; Grant et al., 1989; Roth et al., 1990).

System architecture: The model of system architecture for designing an efficient method of measuring the permittivity of samples in a various frequency range using capacitive sensor is illustrated in Fig. 1.

\section{MATERIALS AND METHODS}

The main aim of our proposed system is to study the capacitance measurements of the liquid samples which are

Corresponding Author: Kalyan Sagar Kadali, Department of EEEM, AMET University, Chennai, India 
affected by frequency. The system uses the capacitance sensor for measuring the complex permittivity of the liquid sample at the certain temperature which is calculated by using temperature sensor. Figure 2 illustrates the block diagram for designing an efficient method of measuring the permittivity of samples in a various frequency range using capacitive sensor. Furthermore, these process can be monitored from anywhere in the world by using the wireless communication.

\section{Hardware components}

Capacitance sensor: All types of metallic and non metallic objects can be identified by using the capacitance sensor. Capacitive sensor can easily detect liquids or glasses which are highly transparent one. Only the relevant thing is the target material with dielectric conductivity. Higher the dielectric constant of a thing, switching distance or detection reliability of the materials will be high. The application of capacitive sensors includes measurements due to high dielectric constant. Capacitive sensors can be able to measure the materials from outside which can occur on certain conditions. These sensors are also employed for identifying solid materials such as wood, plastics and paper. They are available in the most of the sectors in industries because of their protection to all types of factors which interfering it.

Temperature sensor: Temperature sensor is employed in our system for analyzing or measuring the change in capacitance in liquid samples like salt water, tap water and mineral water with the certain temperature.

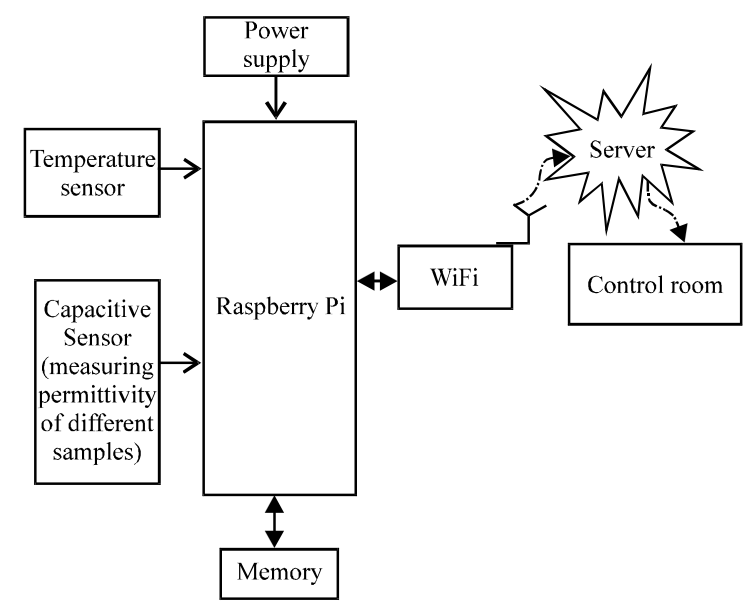

Fig. 2: Block diagram for designing an efficient method of measuring the permittivity of samples in a various frequency range using capacitive sensor
Raspberry Pi 3: Our proposed system uses Raspberry Pi 3 as a central processor. It is employed to control all the process of capacitence sensor and temperature sensor in our system. It is a miniatured computer. It is used in our system because of its reliability and eficiency on different microcontrollers. It only consumes low power. So, it can be functioned over a long period of time in the efficient way.

\section{RESULTS AND DISCUSSION}

The system uses the capacitance sensor for measuring the complex permittivity of the liquid sample at the certain temperature which is calculated by using temperature sensor. Calculated capacitance of different water samples with frequency of $100 \mathrm{~Hz}-2 \mathrm{kHz}$ were illustrated in the Fig. 3.

The results that are obtained from this system show that when the frequency increases, relative complex permittivity will get decreased. Figure 4 illustrates the calculated relative complex permittivity for various liquid samples like salt water, tap water and mineral water with the wide range of frequency.

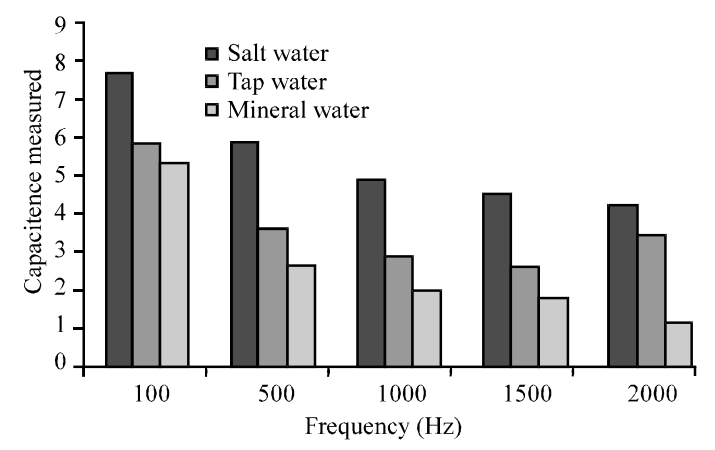

Fig. 3: Calculated capacitance of different water samples with frequency of $100 \mathrm{~Hz}-2 \mathrm{kHz}$

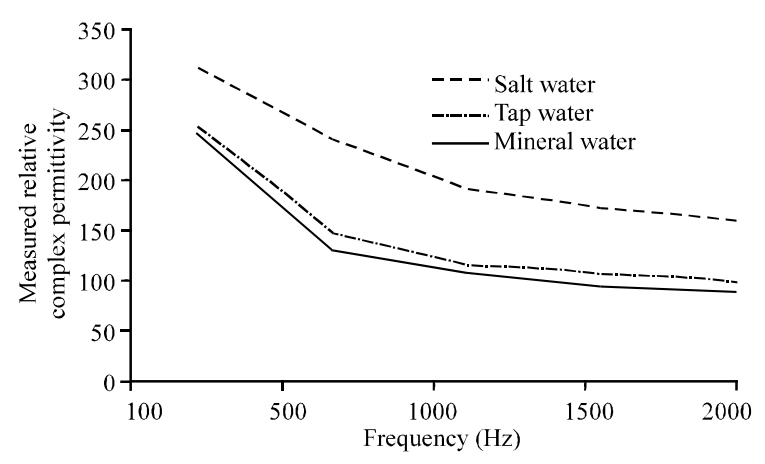

Fig. 4: Calculated relative complex permittivity for various liquid samples with frequency 


\section{CONCLUSION}

An efficient system is developed for measuring the permittivity of the liquid samples in a wide range of frequencies of $100 \mathrm{~Hz}-2 \mathrm{kHz}$ using the capacitive sensor. Measuring of chemical or physical parameters of the materials by changing in the capacitance is known as capacitive sensors. Various methods available for measuring the permittivity, out of those methods, capacitive method are one of the most vital ones. In this study by using cylindrical capacitive sensor and temperature sensor an investigation on the complex permittivity in samples of water can be done.

\section{REFERENCES}

Blackham, D.V. and R.D. Pollard, 1997. An improved technique for permittivity measurements using a coaxial probe. IEEE. Trans. Instrum. Meas., 46: 1093-1099.
Ellison, W., A. Balana, G. Delbos, K. Lamkaouchi and L. Eymard et al., 1998. New permittivity measurements of seawater. Radio Sci., 33: 639-648.

Grant, J.P., R.N. Clarke, G.T. Symm and N.M. Spyrou, 1989. A critical study of the open-ended coaxial line sensor technique for RF and microwave complex permittivity measurements. J. Phys. E. Sci. Instrum., 22: 757-770.

Huang, S.M., A.B. Plaskowski, C.G. Xie and M.S. Beck, 1989. Tomographic imaging of two-component flow using capacitance sensors. J. Phys. E: Sci. Instruments, 22: 173-177.

Krupka, J., K. Derzakowski, B. Riddle and J. Baker-Jarvis, 1998. A dielectric resonator for measurements of complex permittivity of low loss dielectric materials as a function of temperature. J. Meas. Sci. Technol., 9: 1751-1756.

Roth, K., R. Schulin, H. Fluhler and W. Attinger, 1990. Calibration of time domain reflectometry for water content measurement using a composite dielectric approach. Water Resour. Res., 26: 2267-2273. 